\section{Trennung der Chlorisotope durch Thermodiffusion in flüssiger Phase}

\author{
Von Karl F. A lex a nder
}

Deutsche Akademie der Wissenschaften zu Berlin, Institut Miersdorf, Miersdorf/Zeuthen b. Berlin und Rolf Dreyer

z. Zt. Max-Planck-Institut für Chemie, Mainz

(Z. Naturforschg. 10 a, 1034 [1955] ; eingegangen am 12. Oktober 1955)

Ebenso wie im $\mathrm{Gas}$ existiert auch in flüssiger Phase ein Isotopieeffekt der Thermodiffusion, der im Trennrohr nach Clusius und D ickel verstärkt werden kann. Das vorliegende experimentelle Material hierzu ist jedoch noch sehr spärlich.

Korsching und Wirtz erzielten eine geringfügige Trennung der Zinkisotope durch Thermodiffusion in wäßriger Lösung ${ }^{1}$. Die gleichen Autoren untersuchten die Thermodiffusion von $\mathrm{HDO}$ in $\mathrm{H}_{2} \mathrm{O}^{2}$ und von $\mathrm{C}_{6} \mathrm{D}_{6}$ in $\mathrm{C}_{6} \mathrm{H}_{6}{ }^{3}$. F o u r n i e r beobachtete in einem (unglücklich dimensionierten) $3 \mathrm{~m}$ langen Trennrohr mit $2 \mathrm{~mm}$ Wandabstand, das mit Tetrachlorkohlenstoff gefüllt war, eine geringe Verschiebung des Häufigkeitsverhältnisses der Chlorisotope ${ }^{4}$. Schließlich ist bekannt, daß während des Krieges in den USA die Thermodiffusion in flüssigem Uranhexafluorid zur großtechnischen Anreicherung des U 235 versucht wurde ${ }^{5}$.

Da eine kleine, an sich für andere Aufgaben bestimmte Thermodiffusionsapparatur zur Verfügung stand, erschien es uns sinnvoll, diesem Isotopieeffekt nachzugehen und an einem einfachen Beispiel seine Größenordnung zu messen. Die Apparatur wurde bereits an anderer Stelle ausführlich beschrieben ${ }^{6}$. Der ebene Arbeitsspalt hatte die Abmessungen $6 \times 6 \times 0,027 \mathrm{~cm}^{3}$. Oben und unten schlossen sich Vorratsbehälter von je $8,5 \mathrm{~cm}^{3}$ Inhalt an. Die Temperierung erfolgte auf der einen Seite durch Leitungswasser, auf der anderen durch einen Thermostatenumlauf mit $55^{\circ} \mathrm{C}$, so daß der Temperaturunterschied zwischen warmer und kalter Wand etwa $40^{\circ} \mathrm{C}$ betrug.

$\mathrm{Zu}$ den Versuchen wurde Trichloräthylen „reinst“ verwendet, das zuvor in einer 2000/35-Kolonne über Raschig-Ringe $3 \times 3 \mathrm{~mm}$ rektifiziert worden war. Die Isotopieverschiebung wurde nach der Trennung durch relative Dichtemessungen mit Hilfe eines Kapillarpyknometers und einer Analysenwaage bestimmt. Der Inhalt des unteren Trennrohrbehälters hatte nach der Trennung stets eine etwas größere Dichte als der des oberen Behälters. In Vorversuchen wurde die Einstellzeit des Trennrohrs zu etwa $4 \mathrm{~h}$ bestimmt. Daraus läßt sich nach der Trennrohrtheorie ${ }^{7,8}$ der Koeffizient der Selbstdiffusion zu etwa $1,8 \cdot 10^{-5} \mathrm{~cm}^{2} / \mathrm{sec}$ abschätzen.

Die eigentliche Trennung wurde zur Vergrößerung des Effektes in drei Stufen vorgenommen, d. h. die

1 H. Korsching u. K. Wirtz, Abh. Preuß. Akad. Wiss. Nr. 3, 1939.

2 H. Korsching u. K. Wirtz, Naturwiss. 27, 367 [1939].

3 H. K o r s c h in g, Naturwiss. 31, 348 [1943].

4 A. A. Fournier, C. R. Acad. Sci., Paris 215, 529 [1942].

5 H. D. S m y t h, Rev. Mod. Phys 71, 351 [1945]. leichte und die schwere Fraktion der ersten Trennung (die mehrfach durchgeführt wurde, um die notwendige Substanzmenge zu bekommen) wurde später jeweils noch einmal durch das Trennrohr geschickt; in der 3. Stufe wurde dann die leichte Fraktion des leichten Zweiges und die schwere Fraktion des schweren Zweiges der Trennung unterworfen. Für jede Trennrohrfüllung betrug die Trennzeit $4 \mathrm{~h}$.

Zur Kontrolle der Dichtemessungen wurde am Produkt der zweiten Stufe eine Isotopenanalyse durch Aktivierung des $\mathrm{Cl}^{37}$ mit langsamen Neutronen, wie von $\mathrm{H}$ e r r vorgeschlagen und bei $\mathrm{Kl}$ e $\mathrm{mm}$ und $\mathrm{Lund}$ é ${ }^{9}$ angegeben, durchgeführt. Dazu wurde das Trichloräthylen mit Kalium zerstört und das enthaltene Chlor in Form von $\mathrm{AgCl}$ gewonnen. In der leichten Fraktion wurde ein Gehalt von $(23,8 \pm 0,5) \% \mathrm{Cl}^{37}$ und in der schweren Fraktion ein Gehalt von $(25,5 \pm 0,5) \% \mathrm{Cl}^{37}$ gegenüber dem Normalgehalt von $24,6 \%$ festgestellt. Die Umrechnung dieser Werte auf die entsprechende Dichtedifferenz ergibt eine befriedigende Übereinstimmung mit den direkt gemessenen Dichtewerten. In Tab.1 sind die Ergebnisse beider Methoden zusammengestellt.

\begin{tabular}{|c|c|c|}
\hline \multirow{2}{*}{ Stufe } & \multicolumn{2}{|c|}{$(\Delta \varrho / \varrho) \cdot 10^{3}$} \\
& Dichtemessung & Aktivierungsanalyse \\
\hline 1 & $0,5 \pm 0,1$ & \\
2 & $1,15 \pm 0,1$ & $0,8 \pm 0,4$ \\
3 & $1,6 \pm 0,1$ & \\
\hline
\end{tabular}

Tab. 1.

Im Mittel wird pro Stufe das $\mathrm{Cl}^{37}$ um 1,15\% an- bzw. abgereichert. Daraus läßt sich, unter Berücksichtigung der Tatsache, daß die Einstellzeit etwa $4 \mathrm{~h}$ beträgt, mit Hilfe der bekannten Beziehungen der Trennrohrtheorie ${ }^{7,8}$ der Thermodiffusionsfaktor für die Trennung von $\mathrm{Cl}^{35}$ und $\mathrm{Cl}^{37}$ in Trichloräthylen abschätzen. Man erhält

$$
a=1,0 \cdot 10^{-2} .
$$

Dieser Wert hat die gleiche Größenordnung wie die bei isotopen Gasmischungen beobachteten Thermodiffusionsfaktoren (siehe z. B. die Zusammenstellung bei $\mathrm{G} \mathrm{r}$ e w und $\left.\mathrm{Ib} \mathrm{b} \mathrm{s}^{10}\right)$. Die Isotopentrennung durch Thermodiffusion in flüssiger Phase scheint daher mit der Trennung in der Gasphase auch vom praktischen Standpunkt aus durchaus konkurrenzfähig zu sein. Die Versuche werden fortgesetzt.

Der eine von uns (R.D.) dankt Herrn Professor P a neth für die freundliche Einladung zu einem Gastaufenthalt in Mainz. Weiterhin gilt sein Dank Herrn Dr. H e r r (Mainz) für die Anleitung sowie die freundlichen Ratschläge bei der Durchführung der Isotopenhäufigkeitsbestimmung.

${ }^{6}$ K. F. A l ex a n d e r, Z. Phys Chem. 203, 213 [1954].

7 S. R. de Groot, Physica 9, 801 [1942].

${ }^{8}$ R. C. Jo nes u. W. H. F urry, Rev. Mod. Phys. 18, 151 [1946].

${ }_{9}$ A. K le m m u. A. L u n dé n, Z. Naturforschg. 10a, 282 [1955].

$10 \mathrm{~K}$. E. G r e w and T. L. I b b s, Thermal Diffusion in Gases, Cambridge 1952. 